\title{
Dosimetric Comparison of Intensity-Modulated Radiation Therapy and Volumetric Modulated Arc Therapy in Craniospinal Radiotherapy of Childhood
}

\author{
(D) Elif Eda ÖZER, ${ }^{1}$ (D) Yasin ÇOBAN, ${ }^{2}$ (D) Fulya ÇiFTER, ${ }^{3}$ (D) Songül KARAÇAM, ${ }^{2}$ (D Ömer UZEL, ${ }^{2}$ \\ (1) Tahir Sedat TURKAN²
}

'Department of Radiation Oncology, Bakırköy Dr Sadi Konuk Training and Resarch Hospital, İstanbul-Turkey ${ }^{2}$ Department of Radiation Oncology, Cerrahpaşa Faculty of Medicine, İstanbul University, İstanbul-Turkey

${ }^{3}$ Department of Radiation Oncology, Precess Medical Derivatives Inc, Short Hills, New Jersey-USA

\begin{abstract}
OBJECTIVE
Central nervous system (CNS) tumors are the most common solid organ tumors in children. We aimed to compare two modern radiotherapy techniques in target volumes and doses received by organs at risk (OAR).

\section{METHODS}

Eleven patients who had undergone 3D conformal radiotherapy (3DCRT) with the indication of craniospinal radiation therapy (CSRT) were included. OAR and target volumes were defined. The planned target volume (PTV) was PTV of the brain and PTV of the entire spine. A total of $36 \mathrm{~Gy}$ at 1,8 Gy/fraction was given to all patients. Intensity-modulated radiation therapy (IMRT) and volumetric modulated arc therapy (VMAT) plans were prepared.
\end{abstract}

\section{RESULTS}

At mean doses of optic nerve, thyroid, esophagus, heart and oral cavity; VMAT was significantly superior to IMRT. At mean doses of lungs and kidneys; IMRT was better than VMAT. Dmax of VMAT was at lower limits for all OAR. Regarding low doses received by the body, IMRT was better in V2 and V5; while VMAT was better in V15 and V20. For PTV, V95 was $99 \%$ and $97 \%$, and V107 was $2.6 \%$ and $4 \%$ in IMRT and VMAT, respectively. Regarding monitor units (MU), VMAT revealed significantly lower MU than IMRT.

\section{CONCLUSION}

Two techniques are suitable treatment choices for CSRT and may be utilized to diminish the late adverse effects of radiation and to increase disease-free survival rates in patients receiving CSRT. Nevertheless, the risk of secondary cancer development should be kept in mind.

Keywords: Craniospinal radiation therapy; intensity-modulated radiation therapy; volumetric modulated arc therapy. Copyright $\odot$ 2021, Turkish Society for Radiation Oncology

\section{Introduction}

Cancer remains one of the leading causes of death for children all over the world.[1] Leukemia, central ner- vous system (CNS) tumors and lymphomas are the most commonly observed pediatric cancers.[2] Primary malignant tumors of CNS are the most common solid organ tumors in children accounting for
Received: July 11, 2020

Accepted: September 19, 2020

Online: November 27, 2020

Accessible online at:

www.onkder.org

OPEN ACCESS This work is licensed under a Creative Commons

Attribution-NonCommercial 4.0 International License.
PhD. Elif Eda ÖZER

Bakırköy Dr Sadi Konuk Eğitim ve Araştırma Hastanesi,

Radyasyon Onkolojisi Kliniği,

İstanbul-Turkey

E-mail:drelifeda@gmail.com 
approximately $25 \%$ of all cancers in those patients. $[3,4]$ A well-known treatment method for most of these tumors is maximal surgical resection followed by a local or craniospinal radiation therapy. However, there are increasing concerns about the functional status, quality of life and damages left in the tissues and organs of the children undergoing these treatments.

In the early 2000 s, a novel advance for $3 \mathrm{D}$ conformal radiotherapy (3DCRT) has been developed as a result of rapid technologic progress namely Intensity Modulated Radiotherapy (IMRT). IMRT techniques employ various intensities across multiple radiation beams leading to the construction of highly conformal dose distributions, achieved by subdividing each radiation beam into smaller radiation beamlets and varying the individual intensities of these beamlets. [5] After a decade, there has been attempt on arcbased or rotational therapies to overcome some of the limitations of fixed-field IMRT. The basic concept of arc therapy is the delivery of radiation from a continuous rotation of the radiation source and allows the patient to be treated at a full $360^{\circ}$ beam angle. Arc therapies can achieve highly conformal dose distributions and are essentially an alternative form of IMRT.[6] With efficient use of these techniques and good physical planning, the control rate of tumor is enhanced in tumor dose while the toxicity of the normal tissues is not raised.[7] However, the large field of low dose regions leads to some concerns for OAR. Although many studies have been done about the use of IMRT and VMAT in head neck and pelvis tumors, [8] comparative studies for these two modalities in craniospinal therapy are limited.

The purpose of our study is to compare the target volumes and doses received by OARs by re-creating VMAT and IMRT plans in patients who had previously undergone a craniospinal 3D-CRT for curative purposes.

\section{Materials and Methods}

\section{Patients}

Eleven patients treated with the conventional 3D-CRT method for craniospinal radiotherapy were included in the study. The computed tomography (CT) simulations of the patients were re-loaded to the planning system, and IMRT and VMAT plans were carried out by defining OARs and target volumes.

\section{CT Simulation and Treatment Planning}

All patients were immobilized in a supine position using a thermoplastic head and neck mask and with the arms kept in a relaxed position at both sides of the body. In CT simulation images of $2.5 \mathrm{~mm}$ thickness from $2 \mathrm{~cm}$ above the cranial apex to $2 \mathrm{~cm}$ below the termination of the sacrum were sent to the Varian Eclipse treatment planning system (version 8.6) for recontouring the organs at risk and target volumes.

The eyes, optic nerves, thyroid, esophagus, lungs, heart, liver, kidneys, parotids and the oral cavity were contoured as the OARs. Clinical target volume (CTV) of the brain was contoured covering the whole brain with the cribriform plate and the meninges, and CTV of the spinal cord was contoured including the entire spinal canal and subarachnoidal space as seen laterally in T2 weighted magnetic resonance (MR) or CT images from the foramen magnum to the thecal sac. Planned target volume (PTV) was defined as the total of the PTV spinal which is formed by extending the CTV of the brain and spinal for $0.5 \mathrm{~cm}$ in all directions. Dose definition was a total of 36 Gy in 1.8 Gy per fraction for all patients. In target coverage, PTV volume was meant to be at least $95 \%$ and at most $107 \%$ of the prescribed dose. The intention was to protect the OARs as much as possible.

Treatment plans were prepared using the Varian Eclipse planning system (version 8.6) with a progressive resolution optimizer (PRO) and anisotropic analytical algorithm (AAA) so that treatment could be delivered with $6 \mathrm{MV}$ photon energy from Varian iX model linear accelerator (Rapid Arc) which has 120 MLCs and can carry out $\mathrm{kV}$ imaging (CBCT).

\section{VMAT Planning}

Treatment regions were generated by using multiple isocentric methods with $6 \mathrm{MV}$ energy and a maximum dose rate of $600 \mathrm{MU} / \mathrm{min}$. To cover the whole PTV, two full arcs, one between 181-179 degrees in clockwise and the other one between 179-181 degrees counterclockwise directions were used.

Inverse planning module was used with the 5 -phase PRO algorithm that calculates the dose rate, leaf position and gantry rotation rate in a total of 177 control points with $\sim 2$ degree angles to optimize the results Ring control volumes were formed in order to achieve maximum dose conformity and the control dose distribution outside PTV. PTV and OAR dose constraints and dose volume limitations were used as the dose tol- 
erances defined by Radiation Therapy and Oncology Group (RTOG).[9]

\section{IMRT Planning}

Before planning, various gantry angles were examined in order to obtain the most effective dose distribution in the entire craniospinal volume. The best scenario was achieved with $6 \mathrm{MV}$ photon energy by using gantry angles of $90^{\circ}$ and $270^{\circ}$ (two laterals) with forwarding IMRT (field-in-field) technique for the cranial field and five gantry angles $\left(140^{\circ}, 160^{\circ}, 180^{\circ}\right.$, $200^{\circ}, 220^{\circ}$ ) with inverse IMRT technique (sliding window) for the spinal field. The Maximum dose rate was $300 \mathrm{MU} / \mathrm{min}$. Optimal fluencies were converted into real fluency distributions with an anisotropic analytical algorithm (AAA) by using Leaf Motion Calculator (LMC). The dose calculation range was determined as $2.5 \mathrm{~mm}$.

\section{Evaluation of Treatment Plans}

IMRT and VMAT plans were performed in all patients by defining a total dose of $36 \mathrm{~Gy}$ at $1.8 \mathrm{~Gy} /$ fraction. Both treatment methods were compared by using the following definitions: Plans were normalized so that PTV receives at least $95 \%$ of the prescribed dose intending to protect OARs. Each OAR and target volume was evaluated using a dosevolume histogram (DVH). Dosimetric parameters determined for PTV evaluation were PTV mean, D2, D98, V95, V107, V110 in which Vx defined PTV volume including $\mathrm{x} \%$ of the defined dose and Dy dose defined the receiving $y \%$ of the volume.

Plan compatibilities and PTV heterogeneity were calculated with the conformity index (CI) and homogeneity index (HI) formulae determined by RTOG. [10] CI and HI are defined as the ratio of defined dose volume to PTV volume and the ratio of maximum dose to the defined dose, respectively. Mean dose (Dmean) and maximum dose (Dmax) values were separately determined for the OARs. Low dose volume (low dose bath) was also defined in our study using V2, V5, V10, $\mathrm{V} 15$, and V20 volumes of the body. MUs of all plans were calculated and the duration of treatment was determined.

The ethical approval was obtained from the Ethical Committee for Clinical Researches of Cerrahpasa Medical Faculty, Istanbul University, by the decision numbered B.30.2.İST.0.30.90.00719045.

\section{Results}

\section{Demographic Characteristics}

Eleven cases with brain tumors were included in this study. The mean age of the cases at the onset of radiation therapy was $10 \pm 2$ years. Seven of eleven cases (63.6\%) were male. Diagnostic distribution of the patients was medulloblastoma in 6 cases (54.5\%), anaplastic ependymoma in 2 cases (18.2\%), craniospinal extension in 2 cases (18.2\%) and highgrade glioma in 1 case $(9.1 \%)$. A craniospinal radiation therapy of 36 Gy was planned in all cases which had completed their treatments before the study.

\section{Analyses of Dose-volume Histograms}

In all cases, OAR and target volume coverage were compared regarding previously defined criteria. Dmean and Dmax received by the OARs were compared. VMAT was found to be significantly superior to IMRT regarding the optic nerve $(\mathrm{p}=0.0008)$, thyroid $(\mathrm{p}<0.0001)$, esophagus $(\mathrm{p}<0.0001)$, heart $(\mathrm{p}<0.0001)$ and oral cavity $(\mathrm{p}=0.0004)$. IMRT was better at Dmean of the lungs $(\mathrm{p}<0.0001)$ and kidneys $(\mathrm{p}<0.0001)$ than VMAT (Table 1). Dmax of VMAT plan was kept at low limits in all OARs. Doses at all OARs were within the range of clinically acceptable criteria.

When two plans were compared regarding the low dose values received by the body, it was found that IMRT plans were significantly better in V2 and V5, while VMAT plans were significantly better in V15 and V20 $(\mathrm{p}<0.0001)$. No significant result was found for V10. Integral doses were determined by the calculation of the ratio of Body-PTV volume to Body-PTV Dmean, hence, IMRT plans were found to be significantly better than VMAT plan $(\mathrm{p}=0.0011)$ (Table 2$)$.

Target volume coverages were evaluated by obtaining the desired limits in OARs. For PTV, Dmean was $102.7 \%$ in IMRT and $102.3 \%$ in VMAT plan. V95 was higher than the clinically acceptable criteria in both methods, namely $99.7 \%$ in IMRT and $97 \%$ in VMAT plan ( $\mathrm{p}=0.002)$. V107 was found to be $2.6 \%$ in IMRT and $4 \%$ in VMAT plans. D2 and D98 targets, defined by International Commission on Radiation Units and Measurement (ICRU) criteria,[11] were achieved in both VMAT and IMRT plans (Table 2) (Figs. 1, 2).

$\mathrm{CI}$ and $\mathrm{HI}$ values were found to be significantly superior in IMRT plans compared to VMAT $(\mathrm{p}<0.05)$. The number of MU in VMAT was found to be significantly less than IMRT with ( $\mathrm{p}=0.001)$ (Table 3 ). 
Table 1 Comparison of target volume doses at organs at risk between two different planning treatments

\begin{tabular}{ccc}
$\mathbf{n}=11$ & $\begin{array}{c}\text { IMRT } \\
\text { Mean } \pm \text { SD }\end{array}$ & $\begin{array}{c}\text { VMAT } \\
\text { Mean } \pm \text { SD }\end{array}$ \\
\hline
\end{tabular}

\begin{tabular}{|c|c|c|c|}
\hline \multicolumn{4}{|l|}{ Right Eye } \\
\hline $\mathrm{D}_{\text {mean }}$ & $26.03 \pm 8.38$ & $23.27 \pm 2.19$ & 0.143 \\
\hline$D_{\max }$ & $37.7 \pm 0.97$ & $33.07 \pm 2.32$ & 0.001 \\
\hline \multicolumn{4}{|l|}{ Left Eye } \\
\hline $\mathrm{D}_{\text {mean }}$ & $26.46 \pm 5.71$ & $23.81 \pm 2.35$ & 0.087 \\
\hline $\mathrm{D}_{\max }$ & $37.51 \pm 0.69$ & $33.45 \pm 2.53$ & $<0.0001$ \\
\hline \multicolumn{4}{|l|}{ RON } \\
\hline$D_{\text {mean }}$ & $37.0 \pm 0.59$ & $34.96 \pm 1.32$ & 0.0008 \\
\hline $\mathrm{D}_{\max }$ & $37.35 \pm 11.26$ & $36.6 \pm 0.71$ & 0.0056 \\
\hline \multicolumn{4}{|l|}{ LON } \\
\hline$D_{\text {mean }}$ & $37.04 \pm 0.93$ & $34.71 \pm 1.32$ & $<0.0001$ \\
\hline$D_{\max }$ & $37.42 \pm 0.93$ & $36.41 \pm 0.75$ & 0.0118 \\
\hline \multicolumn{4}{|l|}{ Thyroid } \\
\hline $\mathrm{D}_{\text {mean }}$ & $22.94 \pm 3.06$ & $14.37 \pm 1.66$ & $<0.0001$ \\
\hline $\mathrm{D}_{\max }$ & $31.39 \pm 3.32$ & $22.17 \pm 2.2$ & $<0.0001$ \\
\hline \multicolumn{4}{|c|}{ Right Lung } \\
\hline $\mathrm{D}_{\text {mean }}$ & $5.66 \pm 1.13$ & $7.99 \pm 1.25$ & $<0.0001$ \\
\hline $\mathrm{D}_{\max }$ & $32.88 \pm 3.48$ & $28.83 \pm 4.37$ & 0.0044 \\
\hline \multicolumn{4}{|l|}{ Left Lung } \\
\hline $\mathrm{D}_{\text {mean }}$ & $4.43 \pm 1.39$ & $8.1 \pm 1.09$ & $<0.0001$ \\
\hline $\mathrm{D}_{\max }$ & $30.93 \pm 3.5$ & $27.89 \pm 4.19$ & 0.0282 \\
\hline \multicolumn{4}{|l|}{ Heart } \\
\hline $\mathrm{D}_{\text {mean }}$ & $9.13 \pm 1.64$ & $7.06 \pm 1.03$ & $<0.0001$ \\
\hline$D_{\max }$ & $22.53 \pm 3.51$ & $15.03 \pm 2.3$ & $<0.0001$ \\
\hline \multicolumn{4}{|c|}{ Esophagus } \\
\hline $\mathrm{D}_{\text {mean }}$ & $23.72 \pm 3.09$ & $14.68 \pm 1.96$ & $<0.0001$ \\
\hline$D_{\max }$ & $35.88 \pm 1.57$ & $24.6 \pm 3.24$ & $<0.0001$ \\
\hline \multicolumn{4}{|c|}{ Right Kidney } \\
\hline $\mathrm{D}_{\text {mean }}$ & $2.88 \pm 0.91$ & $8.84 \pm 1.51$ & $<0.0001$ \\
\hline$D_{\max }$ & $20.76 \pm 4.93$ & $21.12 \pm 6.13$ & 0.210 \\
\hline \multicolumn{4}{|c|}{ Left Kidney } \\
\hline$D_{\text {mean }}$ & $3.2 \pm 1.33$ & $9.2 \pm 1.65$ & $<0.0001$ \\
\hline$D_{\max }$ & $23.15 \pm 5.8$ & $21.46 \pm 4.8$ & 0.394 \\
\hline \multicolumn{4}{|c|}{ Right Parotid } \\
\hline $\mathrm{D}_{\text {mean }}$ & $15.12 \pm 7.44$ & $12.7 \pm 2.59$ & 0.253 \\
\hline $\mathrm{D}_{\max }$ & $34.25 \pm 4.7$ & $23.87 \pm 5.05$ & 0.001 \\
\hline \multicolumn{4}{|c|}{ Left Parotid } \\
\hline$D_{\text {mean }}$ & $13.77 \pm 7.48$ & $13.67 \pm 3.24$ & 0.963 \\
\hline$D_{\max }$ & $30.89 \pm 8.76$ & $20.87 \pm 5.05$ & 0.037 \\
\hline \multicolumn{4}{|l|}{ Liver } \\
\hline $\mathrm{D}_{\text {mean }}$ & $5.28 \pm 1.25$ & $6.52 \pm 0.92$ & 0.0054 \\
\hline $\mathrm{D}_{\max }$ & $25.8 \pm 3.84$ & $22.03 \pm 4.55$ & 0.0304 \\
\hline \multicolumn{4}{|c|}{ Oral Cavity } \\
\hline$D_{\text {mean }}$ & $14.67 \pm 1.83$ & $9.34 \pm 1.45$ & 0.001 \\
\hline $\mathrm{D}_{\max }$ & $26.0 \pm 3.24$ & $16.22 \pm 1.7$ & $<0.0001$ \\
\hline
\end{tabular}

SD: Standard deviation; IMRT: Intensity-modulated radiation therapy; VMAT: Volumetric modulated arc therapy; RON: Right optic nerve; LON: Left optic nerve; SD: Standard deviation; Vx: Planned target volume including $\mathrm{x} \%$ of the defined dose

\section{Discussion}

The main purpose of the treatment for many CNS tumors in childhood is providing the maximum chance for the cure as well as limiting long term toxicities. CSRT means irradiation of the entire neural axis, including the whole cranium and whole spinal cord until the third sacral vertebra. 3D-CRT is currently a method of choice in CSRT to make better plans by protecting the OARs and minimizing the complication rates with the use of IMRT and VMAT techniques. On the other hand, the greatest advantage of using modern radiotherapy techniques such as VMAT and IMRT is the capability of adjusting high dose areas formed in the overlapping zones with automatic optimization.[12,13] Therefore, in the present study, the target volumes in dosimetric plans and doses received by OARs were compared between IMRT and VMAT, planned in patients who had undergone craniospinal 3D-CRT.

In the study by Fogliata et al., the craniospinal radiotherapy was carried out in 5 cases and VMAT was shown to be superior to conformal radiotherapy regarding the target volume coverage and protection of OAR.[14] Their results VMAT with 36 Gy prescription dose were comparable with our PTV in the mean dose and coverage of target volumes except for V107 being higher than the value in our study.

In the study by Chen et al., VMAT was carried out in the supine position with the rapid arc device and dosimetric results revealed homogenous and conformal dose distribution in the craniospinal field and better protection was acquired for the OAR. They planned CSRT with the VMAT technique for two patients and found that $\mathrm{CI}$ value was 1 , HI value was $12.7 \%$ with D5-D95/Dmean calculation, while D5 was $108 \%$ and D95 was 95\%.[15] In our study, when CI and HI formulae defined by RTOG were used whereas D5 was $106 \%$, D95 was 99\%, CI was 1.21 and HI was 1.13 for IMRT treatment. In VMAT plans, D5 was 106\%, D95 was $97 \%$, CI was 0.9 and HI was 1.19 . Thus, our findings were consistent with the literature when the dose coverage of target volumes was compared taking into account the defined doses.

Al-Wassia et al. performed a comparative analysis of target volume coverage, homogeneity and OAR doses between IMRT and VMAT plans for CSRT. [16] They found that V95 values for PTV in CNS were $98.97 \%$ for VMAT and $99.19 \%$ for IMRT, which were comparable with our results as $97 \%$ for VMAT and $99 \%$ for IMRT, with the coverage in IMRT being slightly higher than Al-Wassia et al. The doses 
Table 2 Comparison of body doses, integral doses and target volume doses between two different planning treatments

\begin{tabular}{lccc}
$\mathbf{n = 1 1}$ & $\begin{array}{c}\text { IMRT } \\
\text { Mean } \pm \text { SD }\end{array}$ & $\begin{array}{c}\text { VMAT } \\
\text { Mean } \pm \text { SD }\end{array}$ & $<0.0001$ \\
\hline Body V2 & $0.51 \pm 0.1$ & $0.8 \pm 0.09$ & $<0.0001$ \\
Body V5 & $0.43 \pm 0.09$ & $0.55 \pm 0.11$ & 0.703 \\
Body V10 & $0.3 \pm 0.08$ & $0.3 \pm 0.09$ & $<0.0001$ \\
Body V15 & $0.233 \pm 0.07$ & $0.21 \pm 0.07$ & $<0.0001$ \\
Body V20 & $0.2 \pm 0.07$ & $0.17 \pm 0.07$ & 0.0011 \\
Integral dose & $128271.01 \pm 47233.02$ & $141448.45 \pm 53147.18$ & 0.2266 \\
D2 & $38.59 \pm 0.32$ & $39.05 \pm 0.86$ & 0.2266 \\
D5 & $38.22 \pm 0.28$ & $38.45 \pm 0.64$ & 0.0014 \\
D95 & $35.92 \pm 0.47$ & $34.92 \pm 0.5$ & 0.001 \\
D98 & $35.216 \pm 0.57$ & $33.74 \pm 0.6$ & 0.0038 \\
V95 & $0.99 \pm 0.01$ & $0.97 \pm 0.01$ & 0.0628 \\
V107 & $0.03 \pm 0.02$ & $0.04 \pm 0.02$ & 0.004 \\
V110 & $0.00045 \pm 0.001$ & $0.01 \pm 0.02$ & \\
\hline
\end{tabular}

IMRT: Intensity-modulated radiation therapy, VMAT: Volumetric modulated arc therapy, SD: Standard deviation, Vx: Planned target volume including $\mathrm{x} \%$ of the defined dose, Dx: dose receiving $\mathrm{y} \%$ of the volume

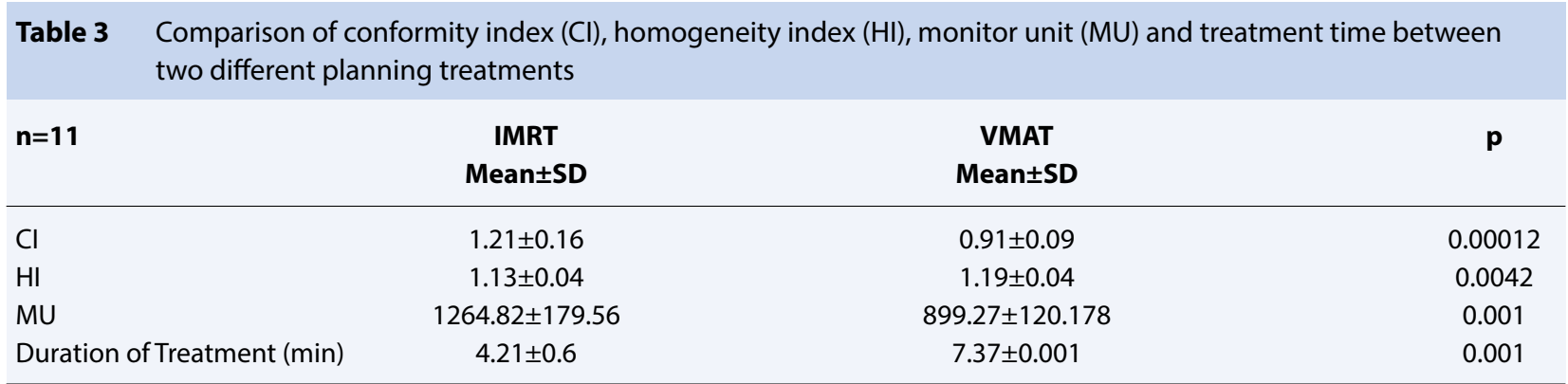

IMRT: Intensity-modulated radiation therapy; VMAT: Volumetric modulated arc therapy; SD: Standard deviation
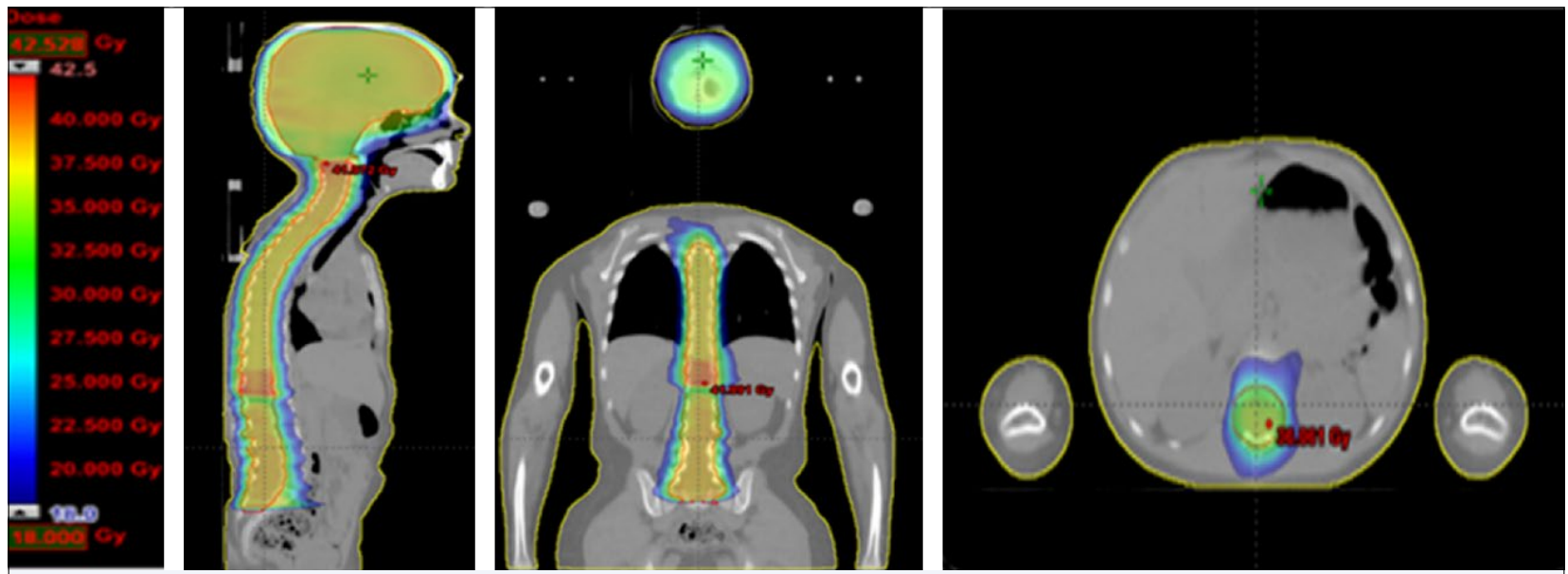

Fig. 1. Isodose distribution of VMAT plan.

to OARs were also found to be slightly lower with VMAT than IMRT,[16] consistent with our findings and most of the literature.[12-15,17]
According to Lee et al., the use of VMAT in CSRT provided protection of radiosensitive organs. However, they claimed that in small organs close to PTV 

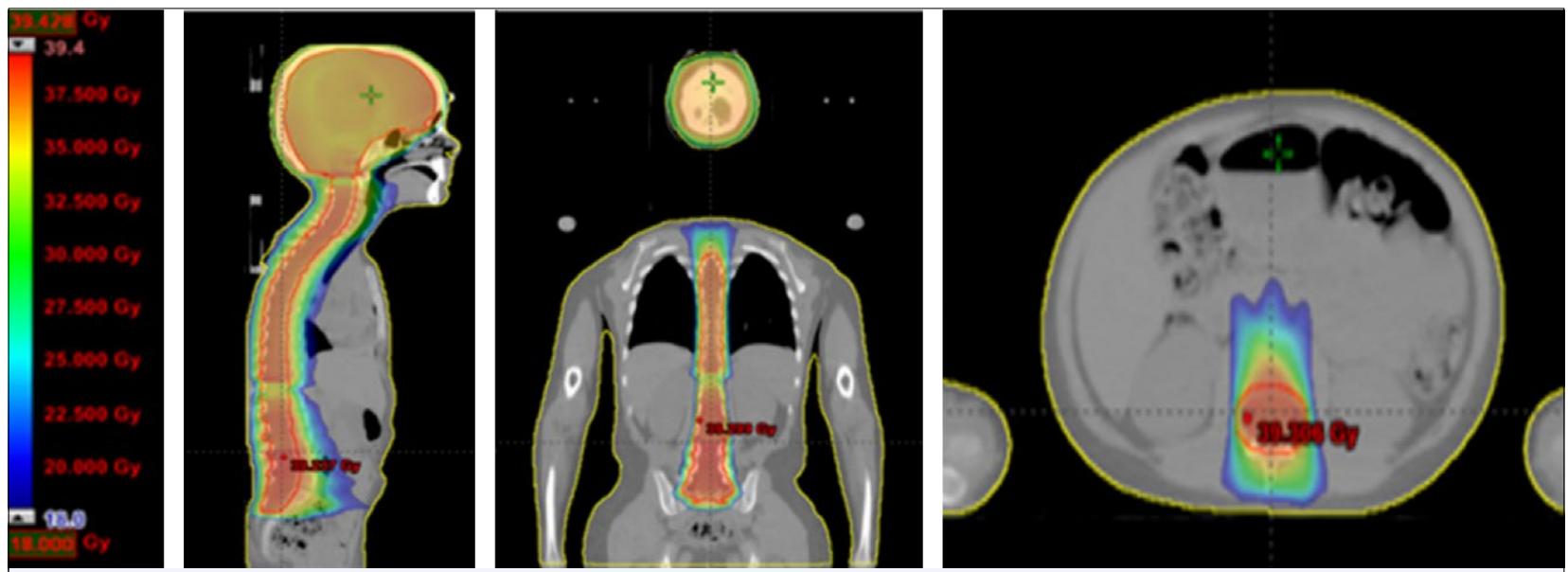

Fig. 2. Isodose distribution of IMRT plan.

or within PTV (cochlea, coronary artery and lens, etc.), low doses could not be obtained in VMAT plans compared with conventional radiotherapy, carrying a potentially increased risk of secondary malignancies. [17] Similarly in our study, the mean doses on optic nerve and eyes doses could not be kept at low limits in both VMAT and IMRT. Lee et al. found that the kidney and liver doses were higher in VMAT plans than conventional methods.[17] In our study, lower doses on kidney and liver were obtained in IMRT plans compared with VMAT. However, for the organs in which doses are critical, optimization can be obtained in lower doses more by reducing the coverage in PTV.

Late-term adverse effects can be minimized by keeping doses on the heart, esophagus, thyroid, liver, and parotids at low limits. The most controversial toxicities in CSRT are cardiac toxicity and a significant amount of exit dose delivered to the heart. In the literature, it is well established that, radiation-induced heart disease and cardiac toxicity are common in children who received craniospinal irradiation.[18,19] In our study, significantly better protection was obtained in the heart, esophagus, thyroid, and parotids with VMAT plans in compliance with the literature.[12-15,17]

The most controversial and unexplored topic in intensity adjusted radiotherapy or in all other multiangled treatments is about the regions receiving low doses. Extension of the field receiving $<10 \mathrm{~Gy}$ and the biological effects of high integral doses are still not clear.[20] However, there are studies reporting that IMRT increases the risk of secondary cancers and malignancies due to extensive high MU in low dose areas.[21] Parker et al. gave a mean of $455 \mathrm{MU}$ in IMRT plans, however, by using the same plans, they calculated a mean of $180 \mathrm{MU}$ with standard techniques in a patient treated with CSRT with a prescription dose of 23.4 Gy. Despite its superiority in protecting OAR, they have stated that the risk of secondary cancer increases with IMRT.[12]

In the study by Miralbell et al., conventional treatments, protons therapies and IMRT were compared regarding the risk of secondary cancers in two patients diagnosed with medulloblastoma and rhabdomyosarcoma using International Commission on Radiologic Protection Model 60. As expected, the risk was least with proton therapy while IMRT was found to be better than conventional therapy.[22] Moreover, in non-craniospinal studies comparing VMAT and IMRT plans, although MU values were found to be $50 \%$ lower in VMAT, they were lower in the low dose receiving volume in IMRT.[23] In our study, VMAT was better than IMRT regarding both protection of the normal tissues and number of MUs delivered, suggesting that VMAT can be more reliable in terms of the risk for secondary cancers in pediatric patients.

While the duration of treatment was $10-15 \mathrm{~min}$ for IMRT and tomotherapy in the study by Parker et al., this was found to be a few minutes for VMAT in the literature.[12,24] However, in our study, the mean duration of treatment was 7-8 min with the 3-zone double arc method and 4-5 min in five-area IMRT. The reason for the shorter duration of treatment in IMRT was that each arc determined as clockwise and counterclockwise in VMAT plans lasted an average of 1.23 $\min$ (Clinac IX). 


\section{Conclusion}

In conclusion, IMRT and VMAT are advantageous treatment alternatives in pediatric patients receiving a CSRT in order to achieve a more homogenous dose distribution and decrease the late adverse effects. Although doses of OARs are significantly lower in the craniospinal axis in VMAT compared with IMRT plan, it is known to increase the integral dose. IMRT is claimed to increase the risk of secondary cancers in the long- term in areas receiving low doses, no randomized studies are showing this. Still, this risk should be taken into consideration and cases should be treated regarding risk-benefit ratios. Comparing VMAT and IMRT plans of previously treated patients after re-structuring, VMAT therapy may provide a better protection in OARs in the CSRT. Randomized controlled phase 3 studies are needed to evaluate the clinical reflection of this result.

Acknowledgement: The present study was presented as publish-only abstract in 2014 ASCO (The American Society of Clinical Oncology) Annual Meeting held in Chicago, Illinois.

Peer-review: Externally peer-reviewed.

Conflict of Interest: None declared.

Ethics Committee Approval: The ethical approval was obtained from the Ethical Committee for Clinical Researches of Cerrahpasa Medical Faculty, Istanbul University, by the decision numbered B.30.2.İST.0.30.90.00719045.

Financial Support: None declared.

Authorship contributions: Concept - E.E.Ö.., T.S.T.; Design - E.E.Ö., T.S.T., Ö.U.; Supervision - E.E.Ö.; Materials - E.E.Ö., Y.Ç.; Data collection \&/or processing - E.E.Ö., Y.Ç.; Analysis and/or interpretation - E.E.Ö., S.K.; Literature search - E.E.Ö., Y.Ç., F.Ç.; Writing - E.E.Ö., F.Ç.; Critical review - E.E.Ö., T.S.T., Ö.U., S.K.

\section{References}

1. Murphy SL, Xu J, Kochanek KD, Arias E. Mortality in the United States, 2017. NCHS Data Brief 2018;328:1-8.

2. Kaatsch P. Epidemiology of childhood cancer. Cancer Treatment Reviews 2010;36(4):277-85.

3. Siegel RL, Miller KD, Jemal A. Cancer statistics, 2018. CA: A Cancer Journal for Clinicians 2018;68(1):730.

4. Ostrom QT, Gittleman H, Liao P, Vecchione-Koval T, Wolinsky Y, Kruchko C, et al. CBTRUS Statistical Re- port: Primary brain and other central nervous system tumors diagnosed in the United States in 2010-2014. Neuro Oncol 2017;19(suppl_5):v1-v88.

5. Webb S. Intensity-modulated radiation therapy using only jaws and a mask. Physics in Medicine \& Biology 2002;47:257-75.

6. Teoh M, Clark CH, Wood K, Whitaker S, Nisbet A. Volumetric modulated arc therapy: a review of current literature and clinical use in practice. The British Journal of Radiology 2011;84(1007):967-96.

7. Purdy JA. Advances in the planning and delivery of radiotherapy: new expectations, new standards of care. Frontiers of Radiation Therapy and Oncology 2011;43:1-28.

8. Palma D, Vollans E, James K, Nakano S, Moiseenko V, Shaffer R, et al. Volumetric modulated arc therapy for delivery of prostate radiotherapy: comparison with intensity-modulated radiotherapy and three-dimensional conformal radiotherapy. Int J Radiat Oncol Biol Phys 2008;72(4):996-1001.

9. Andrews DW, Scott CB, Sperduto PW, Flanders AE, Gaspar LE, Schell MC, et al. Whole brain radiation therapy with or without stereotactic radiosurgery boost for patients with one to three brain metastases: phase III results of the RTOG 9508 randomised trial. Lancet 2004;363(9422):1665-72.

10. Thomas PR, Deutsch M, Kepner JL, Boyett JM, Krischer J, Aronin P, et al. Low-stage medulloblastoma: final analysis of trial comparing standard-dose with reduced-dose neuraxis irradiation. J Clin Oncol 2000;18(16):3004-11.

11. Gregoire V, Mackie TR, De Neve W, Gospodarowicz MK, Purdy JA, van Herk M, et al. Prescribing, recording, and reporting photon-beam intensity-modulated radiation therapy (IMRT): contents. Journal of the International Commission on Radiation Units and Measurements 2010;10(1):1-106.

12. Parker W, Filion E, Roberge D, Freeman CR. Intensity-modulated radiotherapy for craniospinal irradiation: target volume considerations, dose constraints, and competing risks. Int J Radiat Oncol Biol Phys 2007;69(1):251-7.

13. Studenski MT, Shen X, Yu Y, Xiao Y, Shi W, Biswas T, et al. Intensity-modulated radiation therapy and volumetric-modulated arc therapy for adult craniospinal irradiation--a comparison with traditional techniques. Med Dosim 2013;38(1):48-54.

14. Fogliata A, Bergström S, Cafaro I, Clivio A, Cozzi L, Dipasquale G, et al. Cranio-spinal irradiation with volumetric modulated arc therapy: a multi-institutional treatment experience. Radiother Oncol 2011;99(1):79-85.

15. Chen J, Chen C, Atwood TF, Gibbs IC, Soltys SG, Fasola $\mathrm{C}$, et al. Volumetric modulated arc therapy planning method for supine craniospinal irradiation. Jour- 
nal of Radiation Oncology 2012;1:291-7.

16. Al-Wassia RK, Ghassal NM, Naga A, Awad NA, Bahadur YA, Constantinescu C. Optimization of craniospinal irradiation for pediatric medulloblastoma using VMAT and IMRT. Journal of Pediatric Hematology/ Oncology 2015;37(7):e405-e11.

17. Lee YK, Brooks CJ, Bedford JL, Warrington AP, Saran FH. Development and evaluation of multiple isocentric volumetric modulated arc therapy technique for craniospinal axis radiotherapy planning. Int $J$ Radiat Oncol Biol Phys 2012;82(2):1006-12.

18. Stewart JR, Fajardo LF, Gillette SM, Constine LS. Radiation injury to the heart. Int J Radiat Oncol Biol Phys 1995;31(5):1205-11.

19. Jakacki RI, Goldwein JW, Larsen RL, Barber G, Silber JH. Cardiac dysfunction following spinal irradiation during childhood. Journal of Clinical Oncology 1993;11(6):1033-8.

20. Followill D, Geis P, Boyer A. Estimates of whole-body dose equivalent produced by beam intensity modulated conformal therapy. Int J Radiat Oncol Biol Phys 1997;38(3):667-72.

21. Hall EJ, Wuu CS. Radiation-induced second cancers: the impact of 3D-CRT and IMRT. Int J Radiat Oncol Biol Phys 2003;56(1):83-8.

22. Miralbell R, Lomax A, Cella L, Schneider U. Potential reduction of the incidence of radiation-induced second cancers by using proton beams in the treatment of pediatric tumors. Int J Radiat Oncol Biol Phys 2002;54(3):824-9.

23. Cozzi L, Dinshaw KA, Shrivastava SK, Mahantshetty U, Engineer R, Deshpande DD, et al. A treatment planning study comparing volumetric arc modulation with RapidArc and fixed field IMRT for cervix uteri radiotherapy. Radiother Oncol 2008;89(2):180-91.

24. Parker W, Brodeur M, Roberge D, Freeman C. Standard and non-standard craniospinal radiotherapy using helical tomotherapy. Int J Radiat Oncol Biol Phys 2010;77(3):926-31. 